\title{
EchoGéo
}

$46 \mid 2018$

Varia

\section{Les catastrophes cycloniques de septembre 2017 dans la Caraïbe insulaire au prisme de la pauvreté et des fragilités sociétales}

Thierry Nicolas, Françoise Pagney Bénito-Espinal, Émilie Lagahé et MarieLine Gobinddass

\section{(2) OpenEdition}

Journals

Édition électronique

URL : https://journals.openedition.org/echogeo/16439

DOI : 10.4000/echogeo.16439

ISSN : 1963-1197

Éditeur

Pôle de recherche pour l'organisation et la diffusion de l'information géographique (CNRS UMR 8586)

Référence électronique

Thierry Nicolas, Françoise Pagney Bénito-Espinal, Émilie Lagahé et Marie-Line Gobinddass, «Les catastrophes cycloniques de septembre 2017 dans la Caraïbe insulaire au prisme de la pauvreté et des fragilités sociétales », EchoGéo [En ligne], 46 | 2018, mis en ligne le 31 décembre 2018, consulté le 31 juillet 2021. URL : http://journals.openedition.org/echogeo/16439; DOI : https://doi.org/10.4000/ echogeo.16439

Ce document a été généré automatiquement le 31 juillet 2021.

EchoGéo est mis à disposition selon les termes de la licence Creative Commons Attribution - Pas d'Utilisation Commerciale - Pas de Modification 4.0 International (CC BY-NC-ND) 


\section{Les catastrophes cycloniques de septembre 2017 dans la Caraibe insulaire au prisme de la pauvreté et des fragilités sociétales}

Thierry Nicolas, Françoise Pagney Bénito-Espinal, Émilie Lagahé et MarieLine Gobinddass

\section{Introduction}

1 La saison cyclonique 2017 s'est caractérisée par l'atterrissage ${ }^{1}$ de deux ouragans (Irma

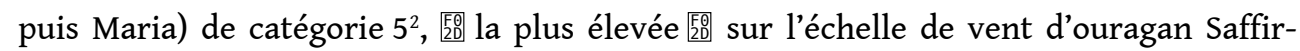
Simpson $^{3}$ en une quinzaine de jours sur la Caraibe insulaire et, entre ces deux perturbations majeures, par la menace de l'ouragan José, de classe 4, quelques jours seulement après l'atterrissage d'Irma. À l'exception du cyclone Andrew qui avait touché les Bahamas en $1992^{4}$, aucun autre ouragan de cette catégorie maximale n'avait frappé les espaces insulaires de la Caraïbe et plus particulièrement les Petites Antilles (tableau 1). Avec des vents moyens soutenus sur une minute à l'impact de l'ordre de 155/160 nœuds, Irma et de Maria sont des phénomènes d'occurrence rare qui se classent parmi les plus puissants du bassin Atlantique Nord.

Tableau 1 - Ouragans du bassin Atlantique Nord, de classe 5, à leur atterrissage ou premiers atterrissages, de 1960 à 2017

\begin{tabular}{|c|c|c|c|c|c|}
\hline Nom & Date & $\begin{array}{l}\text { Pression à } \\
\text { l'impact sur } \\
\text { terre en } \mathrm{hPa} \\
(\mathrm{mb})\end{array}$ & $\begin{array}{lr}\text { Vents } & \text { moyens } \\
\text { soutenus } & \text { sur } \\
1 \text { minute } & \text { à } \\
\text { l'impact } & \text { en } \\
\text { nœuds (kt) } & \end{array}$ & $\begin{array}{l}\text { Zones d'atterrissage } \\
\text { ou premières zones } \\
\text { d'atterrissage }\end{array}$ & Sources \\
\hline
\end{tabular}




\begin{tabular}{|c|c|c|c|c|c|}
\hline Maria & $18 / 9 / 2017$ & 924 & 160 & Dominique & $\begin{array}{l}\text { NHC Public advisory } \\
11 \text { PM AST }\end{array}$ \\
\hline Irma & $6 / 9 / 2017$ & $\begin{array}{l}914-916 \\
915-918\end{array}$ & $\begin{array}{l}160 \text { revu à } 155 \mathrm{kt} \\
160 \text { revu à } 155 \mathrm{kt} \\
\text { sur St-Martin }\end{array}$ & $\begin{array}{l}\text { Barbuda } \\
\text { Saint-Barthélemy } \\
\text { (mur de l'œil) et } \\
\text { Saint-Martin }\end{array}$ & $\begin{array}{l}\text { NHC position } \\
\text { analyse (1), revu par } \\
\text { le NHC, } 2018 \\
\text { NHC r Forecast } \\
\text { Advisory } 30: 15 \text { h00 } \\
\text { UTC, revu par le } \\
\text { NHC, 2018 }\end{array}$ \\
\hline Felix & $4 / 9 / 2007$ & 934 & 140 & Nicaragua & $\begin{array}{lll}\text { NHC } & \text { TCR } & \text { (Felix), } \\
2008 & & \end{array}$ \\
\hline Dean & $21 / 8 / 2007$ & 907 & 150 & Mexique & $\begin{array}{lll}\text { NHC } & \text { TCR } & \text { (Dean), } \\
2008 & & \end{array}$ \\
\hline Andrew & $\begin{array}{l}23 / 8 / 1992 \\
24 / 8 / 1992\end{array}$ & $\begin{array}{l}923 \\
922\end{array}$ & $\begin{array}{l}140 \\
145\end{array}$ & $\begin{array}{l}\text { Bahamas } \\
\text { Floride }\end{array}$ & $\begin{array}{l}\text { NHC Preliminary } \\
\text { Report, H. Andrew, } \\
\text { addendum } 2005\end{array}$ \\
\hline Gilbert & $14 / 9 / 1988$ & 900 & 140 & $\begin{array}{l}\text { Cozumel-Yucatan, } \\
\text { Mexique }\end{array}$ & $\begin{array}{l}\text { NHC, In review and } \\
\text { perspective, } 1991\end{array}$ \\
\hline Edith & 9/9/1971 & ND & 140 & Nicaragua & $\begin{array}{l}\text { Idem, Preliminary } \\
\text { report H. Edith, } \\
\text { NHC, 1971, }\end{array}$ \\
\hline Camille & $17 / 8 / 1969$ & 900 & 165 & $\begin{array}{l}\text { Mississipi, } \\
\text { États-Unis }\end{array}$ & $\begin{array}{l}\text { NHC, In review and } \\
\text { perspective, } 1991\end{array}$ \\
\hline
\end{tabular}

NHC : National Hurricane Center.

TCR : Tropical Cyclone Report.

ND : Non disponible.

(1) Donnée fournie par R. Mazurie, chef de centre Météo-France Montpellier Nîmes.

2 Le caractère exceptionnel d'Irma et Maria 医 qui a été mis en évidence dans deux rapports de la Direction Interrégionale Antilles-Guyane de Météo-France (Degrâce, $2017 \mathrm{a}$ et b) et un article de la Météorologie (Chauvin et al., 2017) [F⿱ vulnérabilité des sociétés insulaires de la Caraïbe. La vision "scientiste» axée sur l'exceptionnalité de ces deux cyclones nous conduit à nous intéresser «à la propension d'éléments exposés comme les biens matériels ou immatériels ainsi que les êtres humains et leurs moyens de subsistance à subir des dommages et des pertes lorsqu'ils sont affectés par des aléas simples ou multiples " (Birkman et al., 2013). Sans verser dans une «interprétation fataliste mettant l'accent sur l'origine "naturelle" de la catastrophe associée à un aléa hydrométéorologique extrême » (Quenault, 2015), nous souhaitons appréhender la capacité différenciée des sociétés insulaires à faire face à un aléa cyclonique en analysant les paramètres qui ont généré l'enchaînement catastrophique du mois de septembre 2017. Loin de s'inscrire dans une tendance atavique qui promeut la «naturalité » des catastrophes (D'Ercole, 2014), il s'agira notamment de questionner la pauvreté qui a été soulignée majoritairement comme un 
facteur de vulnérabilité (Metzger, D'Ercole, 2011) mais aussi d'interroger les structures fondamentales des sociétés insulaires. Une telle approche vise à faire ressortir le fait qu'en dépit du passage d'un ouragan de forte intensité, l'ampleur de la catastrophe peut être différente à l'échelle d'un groupe d'îles proches, voire à l'échelle de la même île divisée par une frontière politique.

\section{Un lourd bilan pour la Caraïbe}

Les passages successifs dans la partie septentrionale de la Caraïbe de deux cyclones de catégorie 5 en une quinzaine de jours ont engendré des pertes très importantes tant sur le plan humain que matériel. En effet, près d'une vingtaine de territoires insulaires, de la Martinique aux Keys, dans le prolongement de la péninsule floridienne, ont été impactés par les effets directs et induits de ces phénomènes, certains territoires ayant même été touchés à deux reprises à l'instar des îles Vierges américaines ou de Porto Rico 5 .

Illustration 1 - Trajectoire et nombre de victimes directes des ouragans majeurs de la saison cyclonique 2017

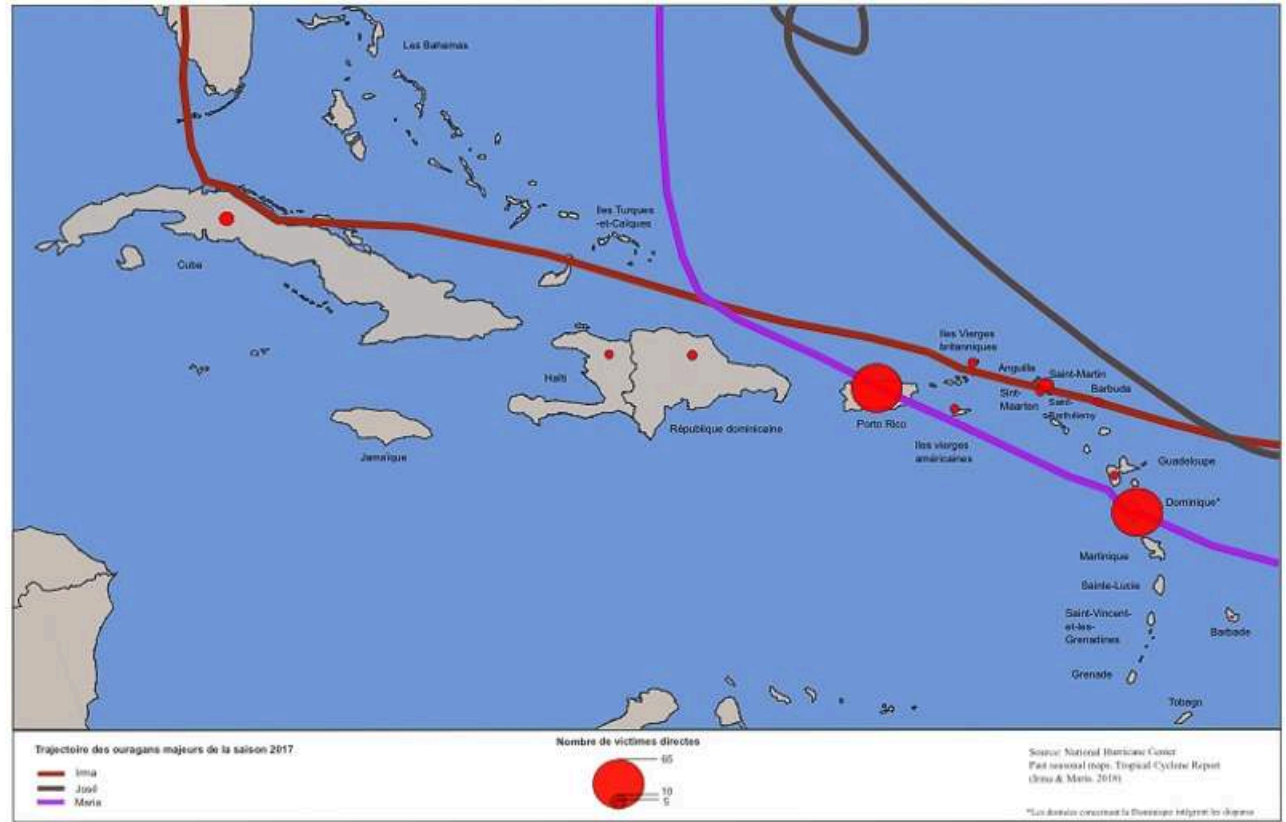

Auteur: T. Nicolas

\section{L'ampleur considérable des dégâts}

Le bilan matériel des deux cyclones se révèle particulièrement lourd dans les territoires insulaires soumis à l'action conjuguée des vents, des précipitations et de la submersion marine. Le coût cumulé de ces passages est évalué à près de 100 milliards d'euros si l'on se fie aux données communiquées par le National Hurricane Center dans ses rapports sur Maria et Irma (NHC, 2018). Dans les îles françaises, « les assurances ont rarement eu à indemniser des biens ayant subi de tels dommages [si bien qu'en] termes de coûts pour l'industrie de l'assurance, Irma est numéro deux sur le podium des sinistres naturels depuis [l'instauration] du régime d'assurance des catastrophes naturelles $»^{6}$. Il faut dire 
que dans ces îles, la quasi-totalité des habitations, des commerces, des bureaux, des structures hôtelières et des bâtiments publics ont été touchés. À Saint-Martin, selon le rapport du délégué interministériel à la reconstruction (Gustin, 2017), la majorité des habitations nécessitaient des travaux de couverture ou de charpente et un peu plus du quart d'entre elles se révélaient dégradées de façon irrémédiable ou présentaient des désordres structurels importants (Illustration 2). De plus, pour de nombreux sinistrés, l'endommagement de leur habitation allait de pair avec des dégâts sur d'autres biens tels que les véhicules ou les embarcations.

\section{Illustration 2 - Un restaurant après le passage d'Irma à Oyster Pond (Saint-Martin)}

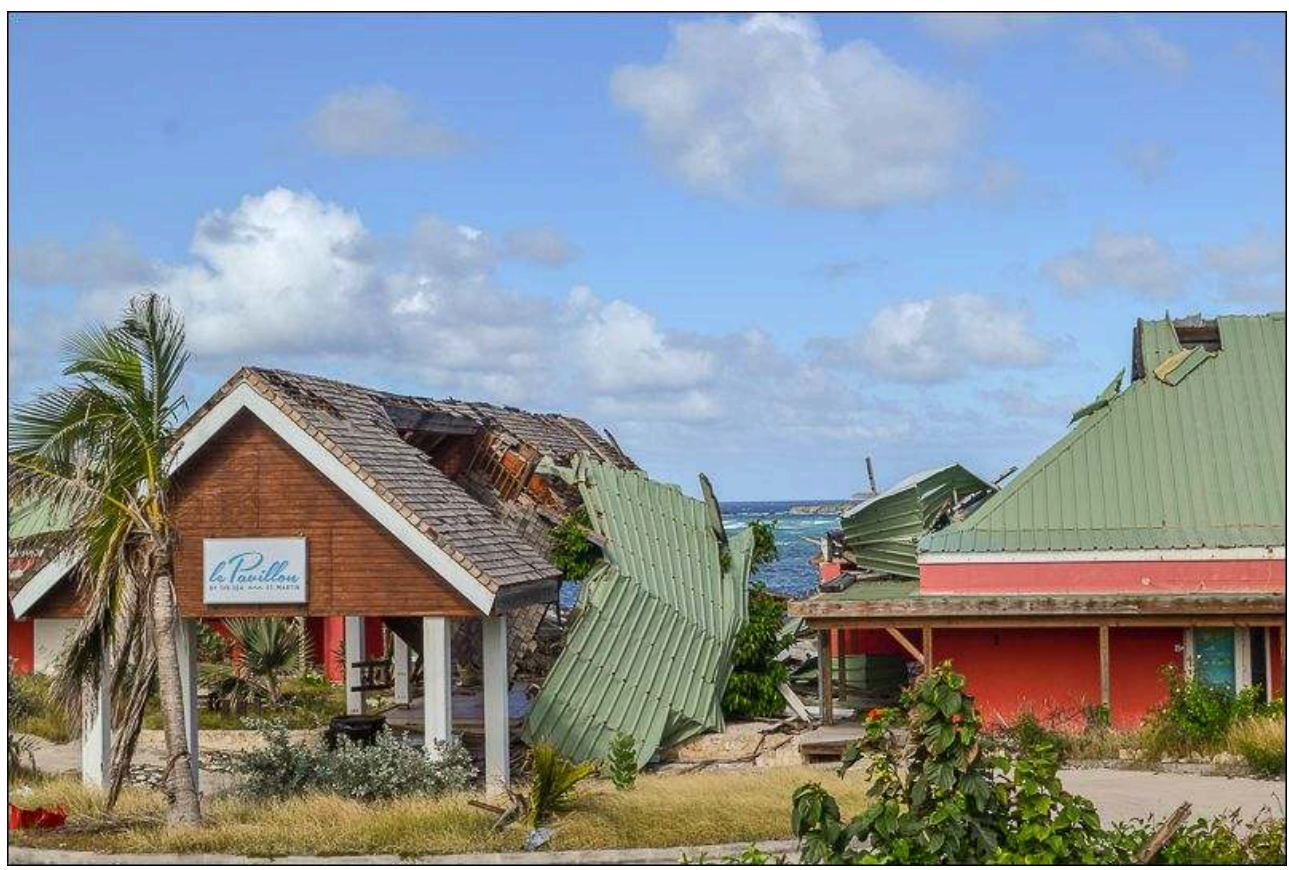

Auteur : T. Nicolas, 23 décembre 2017.

Dans ce contexte, les autorités locales ou nationales ont dû faire face à la nécessité de gérer un amoncellement de déchets de toutes sortes. En dispersant dans l'espace insulaire des biens extrêmement variés, les aléas cycloniques ont contribué à donner aux paysages insulaires, l'image d'un enchevêtrement de débris, éloignée des représentations traditionnellement associées aux îles de la Caraïbe. Aux quatre coins de ces territoires, de véritables «montagnes» d'encombrants sont apparues (illustration 3). Dans la seule partie française de Saint-Martin, ce sont près de 110000 mètres cubes de déchets liés à Irma qui ont été traités sur l'écosite de Grandes Cayes ${ }^{7}$. Le corps du génie de l'armée de terre américaine (USACE) chargé de la collecte à Porto Rico, en a de son côté, ramassé près de 3 millions de mètres cubes ${ }^{8}$ notamment un nombre significatif de tôles froissées et de produits blancs (white goods) tels que des réfrigérateurs et des machines à laver. 
Illustration 3 - Les déchets issus du passage d'Irma à Phillipsburg (Sint Maarten)

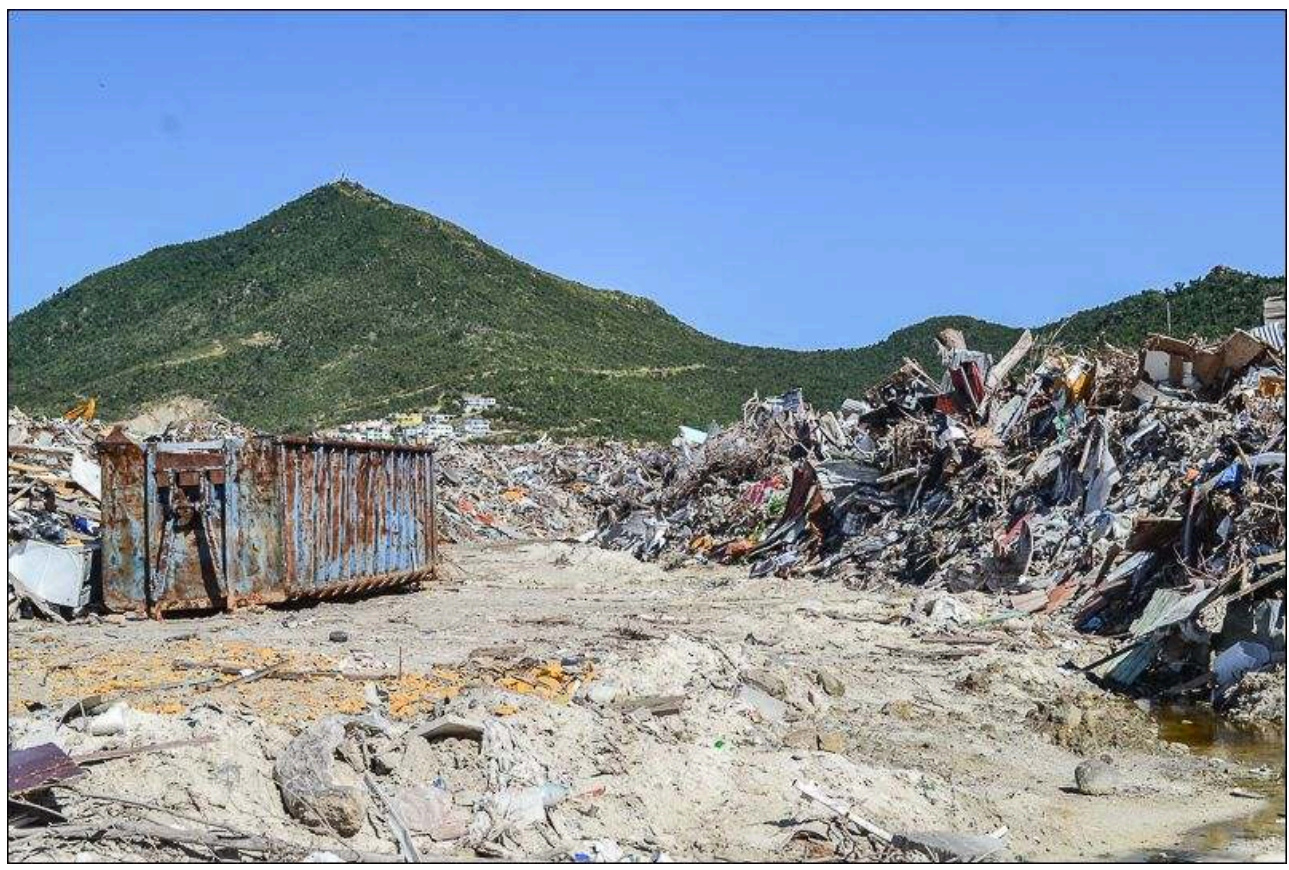

Auteur : T. Nicolas, 23 décembre 2017

6 De nombreux territoires ont connu une interruption du fonctionnement des réseaux d'électricité, de télécommunications ou d'eau potable sans précédent suite à l'enchaînement cyclonique du mois de septembre 2017. Les territoires américains ont été particulièrement touchés surtout en termes d'approvisionnement électrique. Selon des estimations du Groupe Rhodium, une entreprise spécialisée dans les analyses économiques, la privation d'électricité des foyers insulaires américains (Porto Rico et Iles Vierges américaines) par les passages de Maria et d'Irma a eu une durée équivalente à la privation d'électricité de l'ensemble des foyers américains touchés par les cyclones de la décennie 2000, l'ouragan Katrina compris 9 . Le «blackout » lié à Maria constitue désormais le plus important de l'histoire des États-Unis et le second à l'échelle mondiale ${ }^{10}$.

\section{L'impact meurtrier de Maria}

7 Bien que le Président des États-Unis, Donald Trump, ait minimisé l'impact des deux ouragans et en particulier celui de Maria au regard de Katrina ${ }^{11}$ en affirmant que les Portoricains n'avaient pas connu une « vraie catastrophe » et devaient être «fiers » de ne pas comptabiliser des « centaines et des centaines » de morts ${ }^{12}$, il s'avère que le bilan humain est relativement élevé. Douze territoires insulaires différents recensent des victimes directes ou indirectes (illustration 1).

Des deux cyclones qui ont frappé ces territoires, Maria est celui qui s'est avéré le plus meurtrier dans la Caraïbe insulaire. Si seuls six territoires caribéens enregistrent des pertes humaines (Dominique, Guadeloupe, îles Vierges américaines, Porto Rico, Haïti, République dominicaine) suite au passage de Maria, deux d'entre eux concentrent l'essentiel des décès. Porto Rico et la Dominique ont indéniablement payé le plus lourd tribut. L'analyse de la mortalité directe ${ }^{13}$ qui enregistre le nombre de décès à l'issue du 
passage du cyclone et prend généralement la forme de bilans émis par les autorités, en donne une idée. L'État libre associé aux États-Unis enregistre 64 victimes tandis que la Dominique recense depuis la nuit du 18 septembre 2017, près de 31 morts et 34 disparus ${ }^{14}$.

Le bilan de la mortalité directe sur ces deux îles est donc sans commune mesure avec celui des autres territoires balayés par Maria qui varie entre 2 et 5 victimes, ni même avec celui du cyclone Irma. Ce dernier tout en ayant affecté un nombre plus important de territoires en catégorie 5 a causé un nombre de victimes plus faible. Le nombre de décès enregistré dans la Caraïbe insulaire suite au passage d'Irma est inférieur au seul bilan officiel de Porto Rico suite au passage de Maria ${ }^{15}$. On recense en effet moins d'une cinquantaine de victimes depuis l'île de Barbuda jusqu'aux Bahamas avec Irma. Le territoire insulaire le plus touché est l'île franco-néerlandaise du nord des petites Antilles qui dénombre 15 victimes dont 11 pour la seule partie française (Saint-Martin) et 4 pour la partie néerlandaise (Sint. Maarten). Viennent ensuite Cuba (10), les îles Vierges britanniques (4) et américaines (3), puis Porto Rico à nouveau (3), Barbuda (3), Anguilla (1), Haïti (1) et la Barbade (1) ${ }^{16}$.

\section{La pauvreté : la source de vulnérabilité majeure de la Caraïbe?}

10 Parmi les interrogations que soulèvent les bilans des passages successifs d'Irma et de Maria, celle portant sur la vulnérabilité des territoires caribéens en fonction de leur niveau de pauvreté nous apparaît centrale. Nous souhaitons vérifier si, en nous appuyant sur la vulnérabilité physique des personnes, conformément à la littérature existante sur le sujet, les territoires les plus pauvres sont plus vulnérables que les plus riches.

\section{Pauvreté et décès : la Caraïbe comme terrain de comparaison privilégié}

11 Depuis les années 1970, de nombreux auteurs ont souligné le lien particulier qui existe entre la vulnérabilité et le niveau de pauvreté (Baird et al, 1975 ; Hewitt, 1983 ; Blaikie et al. 1994 ; Pelling, 2003, Gaillard et al, 2010). Certains auteurs voient d'ailleurs la pauvreté comme « la première cause profonde de vulnérabilité » (Leone et Vinet, 2006). Les raisons sont à rechercher dans " un habitat de fortune incapable de résister aux éléments naturels, et le plus souvent dans des zones à haut risque (pentes fortes, espaces soumis au débordement des cours d'eau, bords de mer ou de lacs exposés aux inondations et aux houles cycloniques...) » (Pagney Bénito-Espinal, 2003, p. 44). À cela s'ajoutent des facteurs comme l'entassement de plusieurs membres de la famille dans une même habitation ou le manque d'espace entre chaque habitation (Veyret et Reghezza, 2006). Enfin, «le manque de protection sociale ou une protection sociale inadéquate, que ce soit relativement à l'assurance santé, aux services d'éducation, ou encore aux soins hospitaliers conduit à une vulnérabilité d'autant plus accrue » (id.). Cette vulnérabilité des plus pauvres se mesurerait également à l'échelle d'un territoire favorisé où existeraient des poches de pauvreté. L'exemple de l'ouragan Katrina a montré qu'au sein d'un pays riche, les populations les plus démunies restent les plus vulnérables. 
12 L'intérêt d'une approche centrée sur la pauvreté et la vulnérabilité réside dans le fait que la Caraïbe insulaire affectée par Maria et Irma présente, à quelques dizaines de kilomètres de distance, une forte hétérogénéité en matière de richesse. On y retrouve des territoires extrêmement favorisés qui présentent des indicateurs comparables à ceux de pays développés. En termes de richesse par habitant, de nombreux territoires se hissent dans la partie supérieure du classement mondial. À titre d'exemple, dans le classement établi par le World FactBook (CIA), le PIB par habitant en parité de pouvoir d'achat (PPA) des îles Vierges britanniques était en 2014 équivalent à celui de la France $(42300 \$)^{17}$. Certains territoires affichaient même un niveau de richesse supérieur à leur métropole. La partie néerlandaise de Sint. Maarten avec un PIB par habitant (PPA) de 66800 dollars $\left(15^{\mathrm{e}}\right.$ place au niveau mondial) devançait les Pays-Bas $\left(51000 \text { \$, 23 } 3^{\mathrm{e}}\right)^{18}$. De même, selon les statistiques disponibles pour la France produites par le CEROM ${ }^{19}$, Saint-Barthélemy pouvait se prévaloir d'un PIB par habitant (PPA) supérieur à la moyenne nationale. À l'opposé, des territoires caribéens sont situés dans la partie basse du classement mondial à l'image de Cuba, de la Dominique et surtout d'Haïti qui compte parmi les vingt pays les plus pauvres de la planète avec un PIB par habitant (PPA) de $1800 \$ \$^{20}$.

Davantage que le chiffrage des valeurs matérielles endommagées, c'est l'analyse du nombre de décès qui est régulièrement convoquée afin de mettre en évidence cette vulnérabilité différenciée. Car, comme l'affirme L. Boissier, «les décès ne sont pas le résultat du hasard ", mais constituent véritablement « une donnée intégratrice au bout de la chaîne du risque " (Boissier, 2013). Le nombre de morts offrirait la possibilité d'apprécier la vulnérabilité plus générale d'un territoire. La multiplication de bases de données contenant des tables de mortalité (EM-DAT, DesInventar, NatCatSERVICE, Darmouth Flood Observatory, CATNAT...) a encouragé une telle tendance au cours de ces dernières décennies. S'appuyant sur ces données, des instituts de recherche ont établi des indices permettant d'approcher la vulnérabilité. L'IRC (Indice des Risques Climatiques) conçu par Germanwatch est par exemple calibré sur le taux de mortalité lié aux événements météorologiques extrêmes ${ }^{21}$. Cet indice semble confirmer le lien existant entre le niveau de pauvreté et le nombre de décès recensés lors de catastrophes climatiques. Ainsi, sur la période allant de 1996 à 2015, le territoire caribéen ayant connu les pertes humaines les plus importantes est le plus pauvre. La valeur de l'IRC situe Haïti au second rang mondial au cours de cette période. Pour l'année 2016, la partie occidentale de l'île d'Hispaniola occupe la première place à l'échelle mondiale, devançant le Zimbabwe. Le passage du cyclone Matthew en catégorie 4 en octobre 2016 - qui a fait près d'un demi-millier de victimes soit douze fois plus de victimes qu'Irma dans toute la Caraïbe insulaire - explique cette place d'Haïti dans le classement annuel.

\section{L'apparente corrélation entre la pauvreté et le nombre de décès}

Si en 2017, les cyclones Irma et Maria sont passés à distance d'Haïti, tout semble indiquer que les territoires les plus pauvres se sont révélés être les plus vulnérables. Lorsque l'on rapporte le nombre de victimes au nombre d'habitants, on s'aperçoit que les îles les plus affectées par cet enchaînement catastrophique de septembre 2017, sont les plus pauvres. Barbuda, en situation de surinsularité ${ }^{22}$ vis-à-vis d'Antigua, et la Dominique figurent parmi les territoires qui proportionnellement à leur population enregistrent le plus grand nombre de décès (Illustration 4). À ces deux îles, se rajoute, 
en troisième position, Saint-Martin qui à elle seule concentre un quart des victimes liées au passage d'Irma. Bien que n'étant pas considéré stricto sensu comme pauvre, Saint-Martin est l'un des territoires les plus inégalitaires de la région avec des concentrations de populations défavorisées dans des quartiers bien identifiés. La majorité des victimes enregistrées sur terre l'ont été dans les quartiers populaires en particulier ceux de Sandy Ground et Quartier d'Orléans. À bien des égards, on peut considérer que «la vulnérabilité tient à la paupérisation d'une partie non négligeable de cette population qui vit dans des quartiers informels, mal construits où le bâti fragile est soumis aux risques de submersion, d'inondation (Quartier d'Orléans par exemple) et aux vents violents » (Veyret, 2017).

\section{Illustration 4 - Nombre de décès directs pour 10000 habitants suite aux passages d'Irma et de Maria}

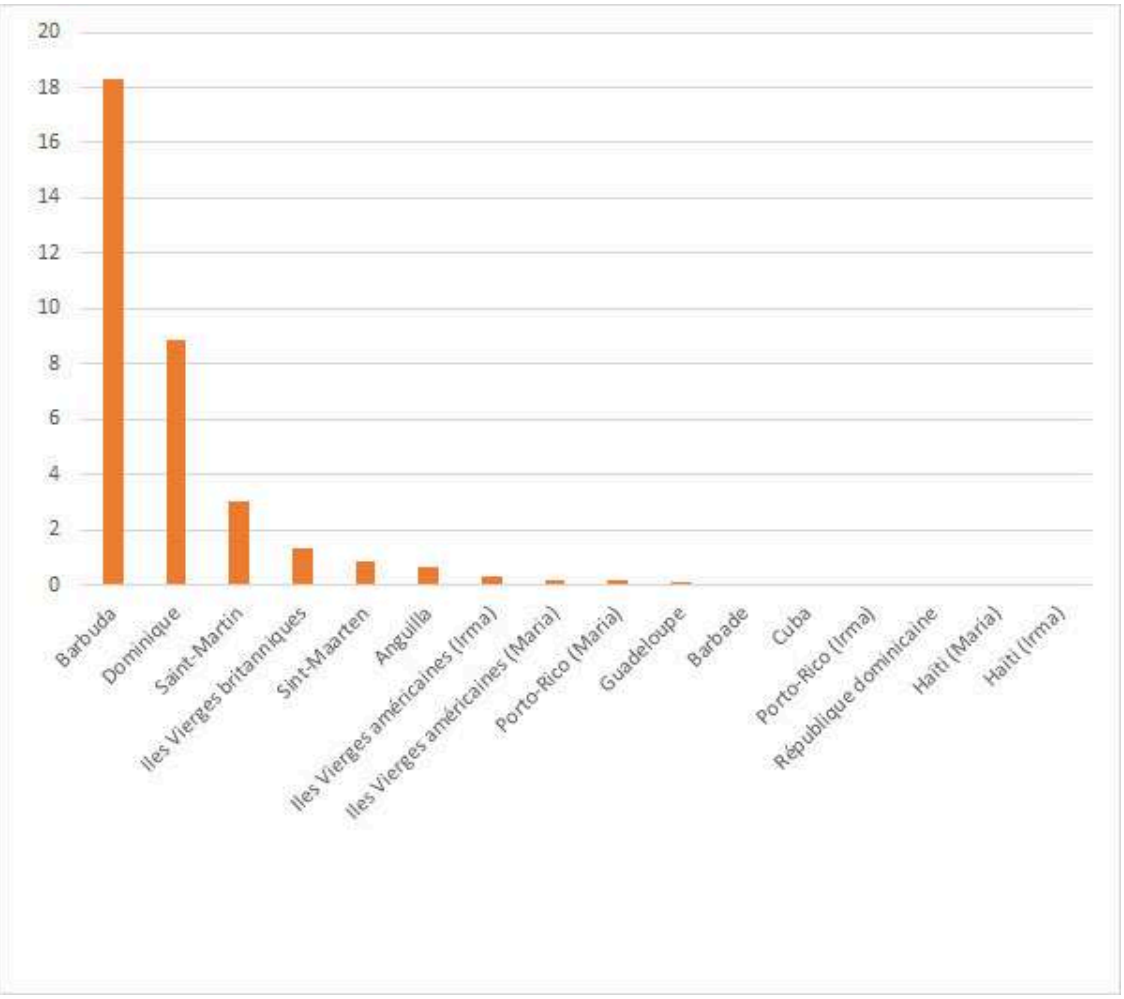

Le bilan officiel de l'île de la Dominique inclut les disparus.

Sources: Tropical Cyclone Report (NHC).

En comparaison, les territoires plus riches enregistrent une plus faible proportion de victimes voire aucune à l'image de Saint-Barthélemy ou des îles Turques-et-Caïques. La qualité des constructions en lien avec les moyens matériels supérieurs des habitants est souvent avancée pour expliquer cette plus faible vulnérabilité humaine. L'analyse de la mortalité directe semble donc valider, en première instance, l'existence d'une corrélation entre la vulnérabilité des habitants et le niveau de pauvreté du territoire.

\section{Le cas cubain : un contre-exemple}

Cependant, lorsque l'on s'intéresse de plus près à la mortalité directe, on s'aperçoit que certains territoires considérés comme pauvres enregistrent un nombre de décès 
relativement faible. Cuba en constitue l'une des illustrations majeures que les saisons cycloniques 2016 et 2017 sont venues confirmer. Lors du passage de Matthew en octobre 2016, la grande île n'a enregistré aucune victime contrairement à Haïti, la République dominicaine ou encore les États-Unis ! Puis en 2017, le passage d'Irma sur treize des quinze provinces du pays (en particulier Camagüey et Ciego de Avila) a occasionné un nombre de victimes relativement limité compte tenu du nombre d'habitants concernés. Cuba affiche, avec dix décès, un bilan inférieur à celui de la partie française de l'île de Saint-Martin.

Cette situation puise notamment ses sources dans l'efficacité du système de réduction $\mathrm{du}$ risque cyclonique mis en place par le régime castriste suite au passage du cyclone Flora, l'un des plus funestes de la Caraïbe qui avait causé près de 8000 morts dans l'arc et environ 1150 à Cuba en octobre 1963 (Pielke et al., 2003). Le gouvernement de Fidel Castro procéda alors à la construction d'un institut météorologique inauguré en octobre 1965 et jeta les bases d'un élargissement de la défense civile au risque environnemental en juillet 1966. Constamment enrichi au fil des décennies et bénéficiant du niveau d'éducation élevé des Cubains (Laguna Cruz et Sanchez Arencibia, 2006), le système s'appuie désormais sur trois piliers. En premier lieu, sur l'organisation d'exercices qualifiés de météores (ejercicios meteoros) au mois de mai, peu avant le début de la saison cyclonique, permettant de répéter les actions à mener en cas de catastrophes $^{23}$. Devenus annuels en 1986, en raison de faiblesses détectées lors du passage de l'ouragan Kate l'année précédente, ces exercices menés sous la houlette du ministère des forces armées et du ministère de l'intérieur ont favorisé l'enracinement d'une culture du risque. Le second pilier consiste à diffuser à la population cubaine, au cours d'une phase informative, des données de qualité sur la trajectoire et les caractéristiques du cyclone (Rubiera, 2014) issues des prévisions conjointes du Centro National de Pronosticos del Instituto de Meteorologia de La Havane (CNPIM) et du National Hurricane Center (NHC) de Miami. Enfin, le troisième pilier repose sur les procédures d'identification et d'évacuation des populations vulnérables. Par l'intermédiaire de nombreux relais locaux, la défense civile encourage les habitants à quitter leur domicile afin de rejoindre des proches disposant d'habitations plus sûres ou des refuges définis à l'avance, généralement des édifices publics. En prévision du passage du cyclone Irma, ce sont près d'un million de Cubains vivant dans des logements précaires ou des zones soumises à des inondations qui ont été déplacés.

18 L'organisation cubaine, bien qu'elle n'épargne pas le territoire de destructions ${ }^{24}$ diminue fortement la vulnérabilité humaine. Ainsi, le coût humain du cyclone Irma, représente moins de $10 \%$ du bilan enregistré lors du passage de Flora pourtant de catégorie inférieure (catégorie 3).

\section{Les îles de l'avant et de l'après : les territoires défaillants en matière de réduction du risque cyclonique}

19 Si l'enchaînement cyclonique du mois de septembre 2017 ne permet pas de mettre en évidence un lien net entre la pauvreté des territoires caribéens et les bilans humains des catastrophes, il donne en revanche la possibilité d'identifier des territoires défaillants en matière de réduction du risque cyclonique. Ces territoires ne sont pas 
uniformes quant à leur niveau de richesse et de développement, leur configuration insulaire ou leur degré de dépendance vis-à-vis d'une métropole. Ils présentent la caractéristique commune d'avoir connu une crise sans précédent suite au passage d'Irma ou de Maria. Pour quatre territoires, Barbuda, la Dominique, Saint-Martin et Porto Rico, ces cyclones ont révélé de profondes carences dans la capacité à gérer un phénomène cyclonique intense. Le cas de Saint-Martin s'apparente à un cas d'école. La partie française de l'île binationale cumule un certain nombre de faiblesses qui ont donné un caractère désastreux au passage d'Irma. L'un des principaux éléments avancés tient à la problématique de l'aménagement du territoire. En effet, de nombreuses constructions ont vu le jour sur le littoral depuis la fin des années 1980 notamment sur la fameuse bande des cinquante pas géométriques pourtant considérée comme une propriété de l'État. Cette dynamique a été facilitée par une forme de permissivité et les insuffisances des documents réglementaires produits depuis les années 1990 (Plan d'Occupation des Sols, Plan de Prévention des Risques Naturels...).

Toutefois, l'ampleur de la catastrophe ne peut se résumer au caractère anarchique de l'occupation et de l'aménagement du territoire saint-martinois. Parmi les facteurs explicatifs, les caractéristiques de la population saint-martinoise sont fondamentales. En effet, celle-ci présente un aspect composite [50 résultant de vagues migratoires importantes initiées à la fin des années 1980 par le développement du tourisme 緊 qui a eu pour effet de favoriser une dilution de la mémoire du risque. En effet, le caractère récent de la majeure partie de la population de Saint-Martin (Duvat, 2008) n'a pas permis la conservation et la transmission de la mémoire du risque cyclonique. Virginie Duvat le notait déjà suite au passage du cyclone Luis en évoquant le fait que les personnes immigrées s'étaient avérées particulièrement vulnérables (id.).

21 L'aspect composite de la population saint-martinoise qui se traduit par la présence de plus d'une centaine de nationalités pour une population d'environ 30000 habitants n'a pas favorisé, par ailleurs, la mise en place de réseaux de solidarité de grande ampleur d'autant qu'à cette fragmentation par origine se superpose une forte disparité des niveaux de vie. Cette situation aboutit à une ségrégation sociospatiale qui se manifeste par l'existence aux côtés de quartiers dégradés, de zones de logements pavillonnaires et de communautés fermées de haut standing (illustration 5). Ce faisant, lorsque la solidarité existe, elle s'exprime le plus souvent entre les individus qui se sentent appartenir à la même communauté et/ou à la même classe sociale. 


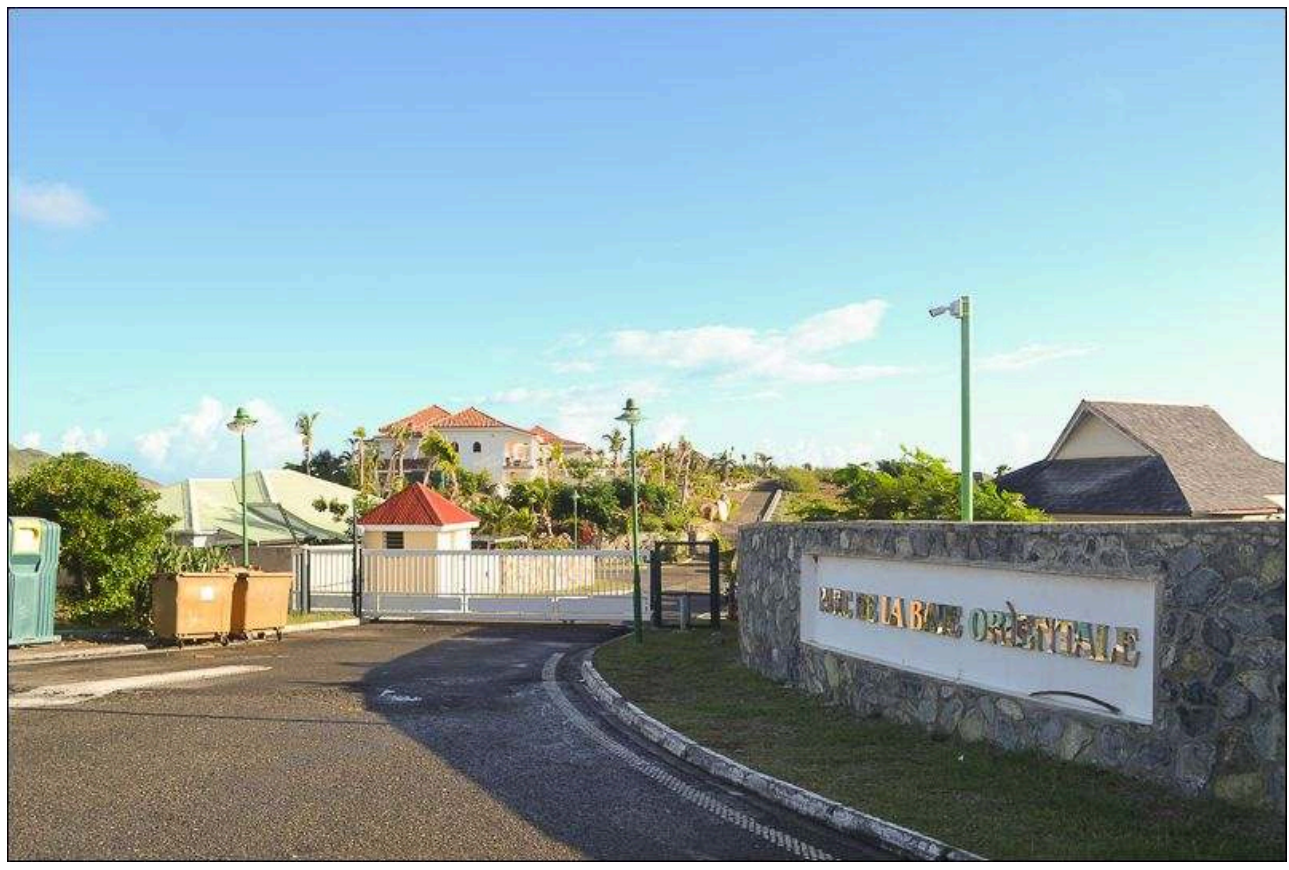

Auteur : T. Nicolas, 24 décembre 2017

En outre, il apparaît qu'à chaque étape de la crise, de nombreuses défaillances sont apparues. Avant le passage du cyclone, en dépit de la qualité de l'alerte et de l'information des habitants, les procédures d'évacuation des populations vulnérables sur terre comme sur mer ont rapidement montré leurs limites. De nombreux habitants ont préféré rester dans leur habitation ou sur leur embarcation. Il faut dire que des doutes existaient quant à la capacité de résistance de certains abris considérés comme sûrs. Il en va ainsi du collège Soualiga qui, jusqu'à la veille de l'atterrissage d'Irma (5 septembre 2017), était présenté comme un refuge supplémentaire de 150 places avant d'être au dernier moment retiré de la liste des centres d'hébergement d'urgence. Ce retrait bien qu'il ait été salvateur au vu de l'état de destruction du collège (illustration 6), révèle surtout le degré d'impréparation lié à l'accueil des personnes potentiellement vulnérables à l'approche d'un aléa cyclonique de forte intensité.

Extrait du communiqué de presse de la préfète déléguée de Saint-Barthélemy et de Saint-Martin du 5 septembre 2017 à 9 h40 :

«Pour les personnes n'ayant nul endroit où se mettre en sécurité, 2 centres d'hébergement d'urgence supplémentaires seront ouverts à Saint-Martin :

- collège Soualiga de Cul de Sac (150 places)

- cité scolaire de la Savane (500 places)

Les personnes qui rejoignent ces centres d'hébergement d'urgence ou les églises sont invitées à se munir d'un peu de nourriture, d'eau, de médicaments et de quoi occuper les enfants.

Ces centres d'hébergement seront ouverts à partir de $11 \mathrm{~h}$ : rejoignez-les !

[...] À compter de ce midi, des bus seront mis à disposition pour aider les personnes qui n'ont pas de véhicule particulier, pour aider à rejoindre les centres d'hébergement de la cité scolaire et du collège Soualiga. » 
Illustration 6 - Un centre d'hébergement d'urgence (collège Soualiga) durement touché par le passage d'Irma

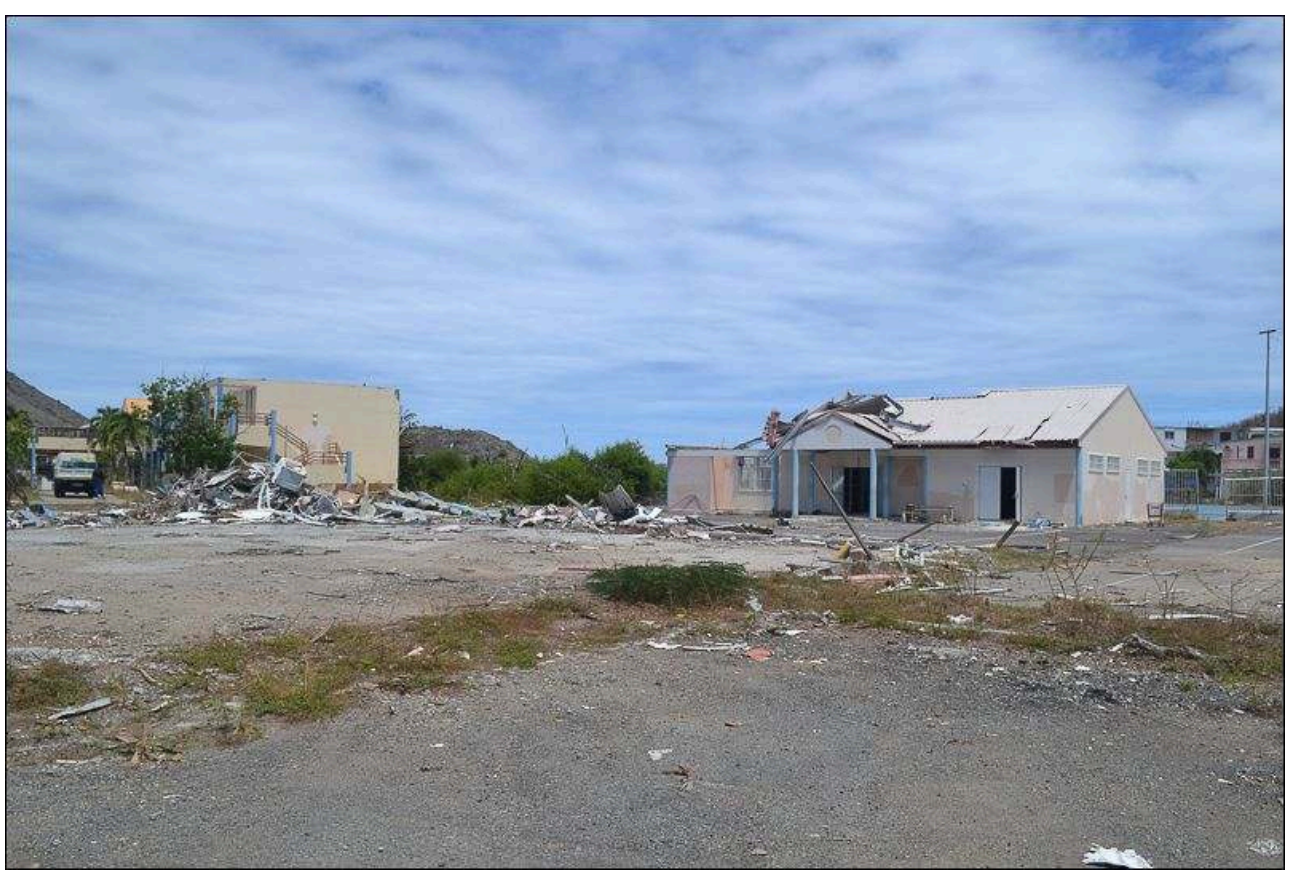

Auteur : T. Nicolas, 27 août 2018.

En comparaison, à Cuba, les procédures d'évacuation sont en général suivies par les habitants car elles peuvent avoir un caractère contraignant. De plus, la sécurisation des domiciles et l'existence d'un réseau d'accueil de proximité largement éprouvé poussent les personnes évacuées à quitter plus facilement leur domicile (Pichler et Striessnig, 2013).

L'ampleur de la catastrophe s'explique également par le fait que, pendant le passage du cyclone, les services de l'État et les secours se sont montrés déficients. La préfecture (illustration 7) a été rapidement détruite, des casernes de pompiers et de gendarmerie ont été fortement touchées de même que l'hôpital de Marigot. Affairés à assurer et garantir leur propre protection, les personnels chargés de gérer la crise n'ont pas pu être aussi disponibles que le nécessitait la situation. Le rapport Gustin témoigne, par exemple, des conditions dantesques dans lesquelles s'est retrouvé le personnel de la préfecture au point que plusieurs mois après, certains de ses agents restaient « très affectés par l'épreuve vécue » (Gustin, 2017). 
Illustration 7 - La préfecture lourdement affectée (Saint-Martin)

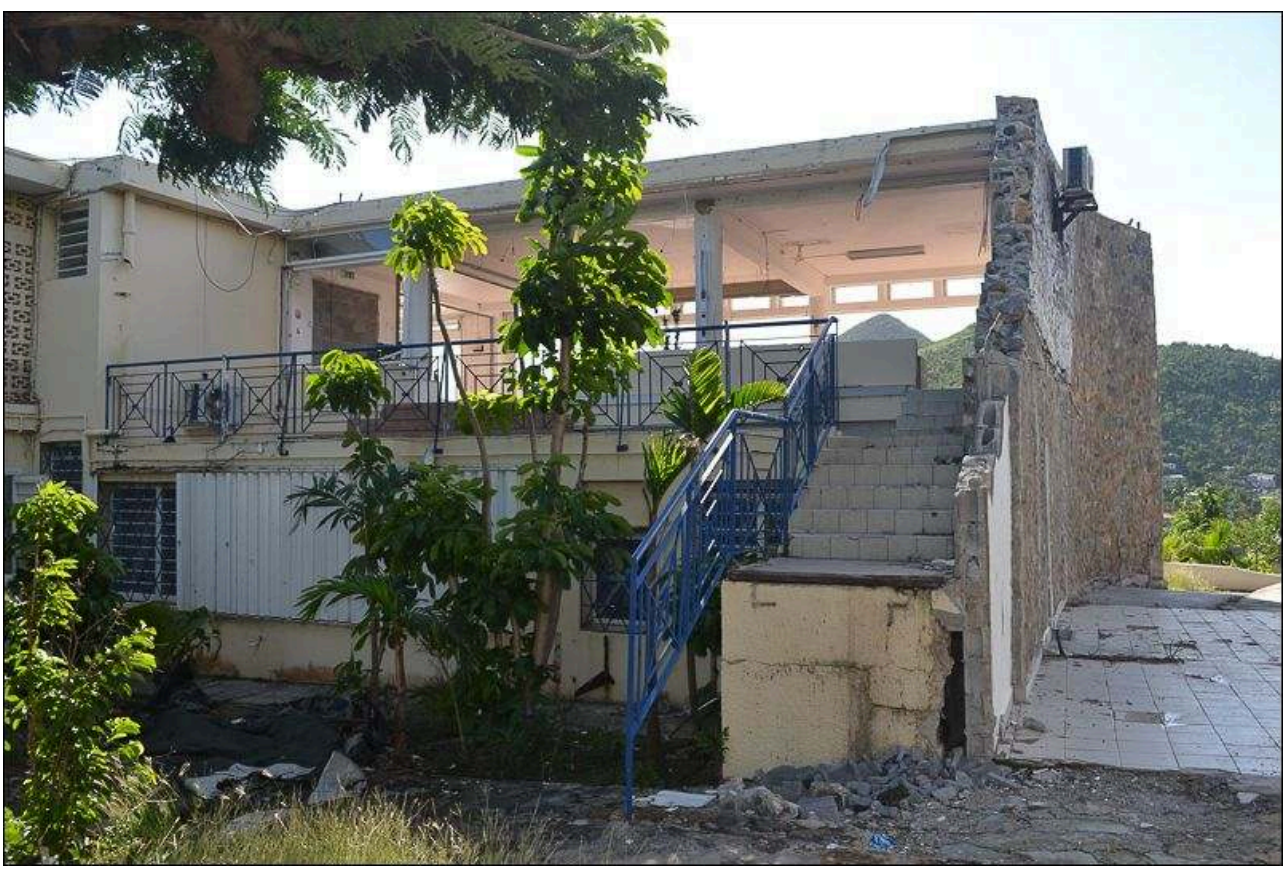

Auteur : T. Nicolas, 24 décembre 2017.

La gestion de l'après-Irma s'est révélée aussi extrêmement compliquée dans la partie française de Saint-Martin. C'est notamment la multiplication des pillages et l'émergence d'un sentiment d'insécurité qui ont rendu la crise encore plus dramatique. De nombreux magasins, des structures hôtelières ou encore des résidences ont été dévalisés et mis à sac (illustrations 8 et 9) ${ }^{25}$. Le retour tardif à la stabilité couplé à la multiplication de rumeurs notamment sur l'évasion de prisonniers armés dans la partie hollandaise, le départ de la préfète ont plongé une partie de la population dans une profonde détresse. Mal informés, en l'absence d'infrastructures de télécommunications et de médias surtout radiophoniques, de nombreux habitants ont rapidement exprimé le souhait de quitter une île qui à leurs yeux sombrait dans l'apocalypse. Ce sont en effet près de 8000 personnes qui ont choisi de quitter l'île temporairement ou définitivement après le passage du cyclone Irma. 
Illustration 8 - Le pillage d'une structure hôtelière à la baie orientale (Saint-Martin)

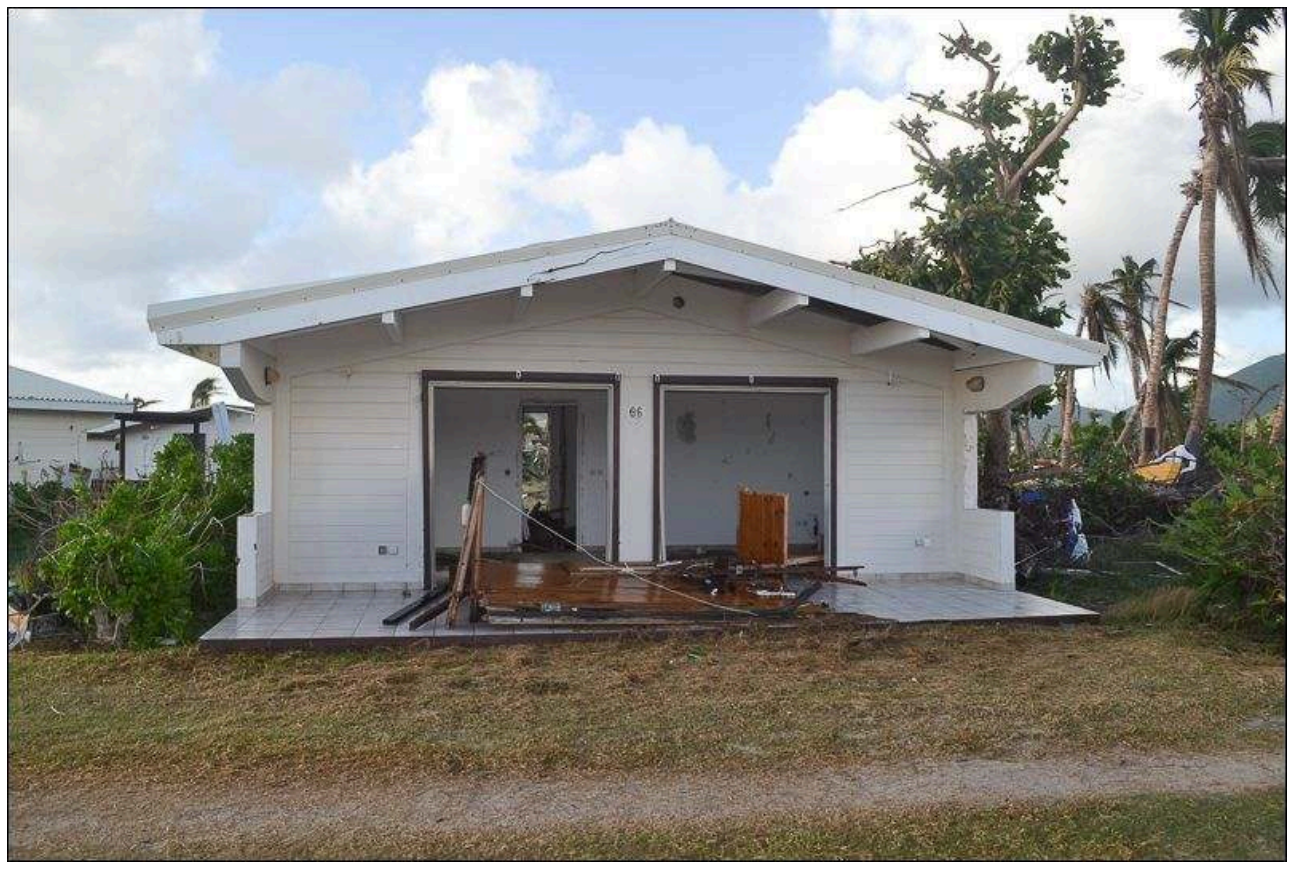

Auteur : T. Nicolas, 23 décembre 2017

Illustration 9 - Le pillage d'un magasin de téléphonie mobile de la ZAC Hope Estate (Saint-Martin)

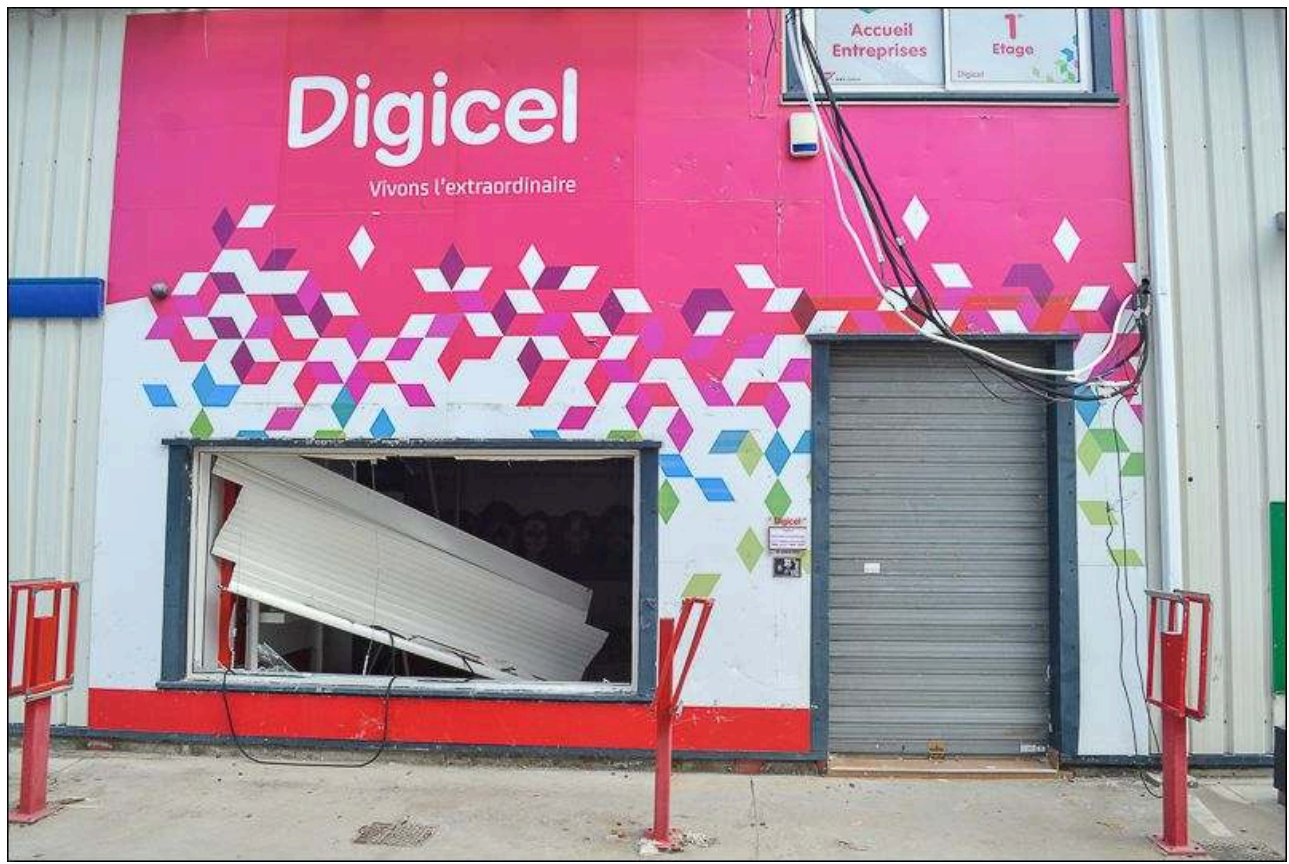

Auteur : T. Nicolas, 23 décembre 2017

Les dégâts provoqués par le cyclone et les conditions de retour du territoire à une forme d'équilibre ont également généré à Porto Rico des flux significatifs de migrants. Plusieurs centaines de milliers de Portoricains ont opté pour d'autres cieux à leurs yeux plus cléments en particulier celui de la Floride. Pour les chercheurs du Centre d'études portoricaines du Hunter College de l'Université de New-York (CUNY), l'île devrait 
perdre au total 470000 habitants soit l'équivalent du nombre de départs durant la décennie précédant le passage du cyclone Maria (Meléndez et Hinojosa, 2017). L'État libre associé aux États-Unis verrait ainsi sa population baisser de 14 \%. À Barbuda, bien que le départ de l'ensemble des habitants de l'île ait été décidé par le gouvernement d'Antigua en prévision du passage du cyclone José, il s'avère qu'une bonne partie de ces déplacés ne souhaitent plus y retourner.

La grande majorité des territoires défaillants voient donc une partie non négligeable de leur population vivre de manière provisoire ou permanente hors de leurs frontières ou des limites de leur circonscription de résidence. L'ampleur du traumatisme lié au passage d'Irma ou de Maria et les conditions dans lesquelles la crise a été gérée, ont créé un environnement propice à l'émergence de nouvelles migrations. La nouveauté réside dans le fait que celles-ci semblent désormais indépendantes à la fois du niveau de richesse du territoire considéré, du degré d'intégration à une mère-patrie ou de la configuration insulaire.

\section{Conclusion}

L'analyse des impacts d'Irma et de Maria sur la Caraïbe montre qu'il faut éviter de proposer des corrélations simplistes entre l'intensité maximale des ouragans observés, la vulnérabilité et leur impact catastrophique. L'exemple d'Irma démontre que l'atterrissage d'un cyclone très puissant n'engendre pas systématiquement un nombre élevé de victimes. Avec moins de cinquante victimes directes dans l'ensemble de l'arc insulaire, Irma ne figure pas parmi les cyclones les plus meurtriers. En réalité, l'analyse de la vulnérabilité insulaire à un aléa intense ne doit pas se limiter à la prise en compte du niveau de richesse, du statut politique ou de la configuration insulaire. Il est nécessaire de mieux appréhender le degré de préparation ou d'impréparation des populations à faire face au risque à travers l'analyse des politiques de réduction du risque cyclonique et de l'effectivité de leur mise en pratique ou des fragilités inhérentes à chacune des sociétés insulaires. Trop souvent ignorée, la politique menée depuis plus d'un demi-siècle par Cuba [50 qui apparaît aujourd'hui comme une illustration des principes préconisés dans le cadre d'action de Sendai - témoigne par exemple de la nécessité de délier la vulnérabilité de la pauvreté que le prisme haïtien pourrait à lui seul valider. De même, les dysfonctionnements enregistrés à Saint-Martin (partie française de l'île) ou à Porto Rico, invitent à repenser le lien entre l'appartenance à une grande nation et le niveau de vulnérabilité, et à s'intéresser aux maux sociétaux qui conduisent à la catastrophe. Pour ces territoires qui se sont révélés défaillants, l'enjeu consiste d'une part comme le rappellent Sandrine Revet et Julien Langumier (2013) à "savoir discerner et rendre compte non plus de ce que la catastrophe détruit, mais bien de ce qu'elle contribue à produire, à faire advenir comme recompositions sociales ». D'autre part, il s'agit de s'interroger sur la nouvelle injonction promue par les autorités locales ou nationales d'une reconstruction durable (Gustin, 2017). Tant à Saint-Martin qu'à Porto Rico, la volonté de tendre vers une meilleure reconstruction se heurte à la réalité du terrain. En effet, si le discours de la "reconstruction durable " apparaît comme une démarche rationnalisée et difficilement contestable, il n'en demeure pas moins que les réponses observées sur le terrain largement marquées du sceau de la "débrouillardise » et de l'impatience sont difficilement conciliables avec une volonté institutionnelle de trouver des solutions innovantes et pérennes. 


\section{BIBLIOGRAPHIE}

Baird, A., O'Keefe, P., Westgate, K., Wisner, B. 1975. Toward an Explanation of Disaster Proneness, Disaster Research Unit. University of Bradford, 52 p.

Birkmann J., Cardona O.D., Carreño M.L., Barbat A. H., Pelling M., Schneiderbauer S., Kienberger S., Keiler S.M., Alexander M., P. Zeil P., Welle T., 2013. Framing vulnerability, risk and societal responses: the MOVE framework. Natural Hazards [En ligne], vol. 67, n², p. 193-211. https:// doi.org/10.1007/s11069-013-0558-5

Blaikie, P., Cannon, T., Davis, I., Wisner, B. (dir.) 1994 (1ère édition), At risk: natural hazards, people's vulnerability, and disasters. Londres, Routledge, $134 \mathrm{p}$.

Boissier L., 2013. La mortalité liée aux crues torrentielles dans le Sud de la France: une approche de la vulnérabilité humaine face à l'inondation. Thèse de doctorat, Université Paul Valéry, Montpellier 3, $212 \mathrm{p}$.

Cangialosi J.P., Latto A. S., Berg R. 2018. Hurricane Irma (AL112017). 30 August-12 September 2017. National Hurricane Center Tropical cyclone Report, 111 p.

Chauvin F., Degrâce J.N., Kapikian E., Montout C., Soubeyroux J.M., 2017. Une saison cyclonique 2017 particulièrement dévastatrice pour les Antilles. La Météorologie, nº 99, p. 3-4.

Degrâce J.-N., 2017a. Passage de l'ouragan exceptionnel Irma sur les îles françaises des Antilles les 5 et 6 septembre 2017. Météo-France, Direction Interrégionale Antilles-Guyane, 6 p.

Degrâce J.-N., 2017b. Passage de l'ouragan exceptionnel Maria sur les îles françaises des Antilles les 18 et 19 septembre 2017. Météo-France, Direction Interrégionale Antilles-Guyane, avec l'aide des centres de Martinique, de Guadeloupe et de la Division prévision, 13p.

Degrâce J.N., avec la contribution de Gilles Lavogiez, 2018. Saison cyclonique 2017, synthèse chiffrée. Direction Interrégionale Antilles-Guyane, Météo-France, 4 p.

D’Ercole R., 2014. Vulnérabilité : vers un concept opérationnel ? (Conférence invitée). Colloque international Connaissance et compréhension des risques côtiers : aléas, enjeux, représentations, gestion. Brest, Institut Universitaire Européen de la Mer (3-4 juillet).

Duvat V., 2008. Le système du risque à Saint-Martin (Petites Antilles françaises), Développement durable et Territoires, Dossier 11 [En ligne]. https://journals.openedition.org/ developpementdurable/7303

Gaillard J.-C., Wisner B., Benouar D., Cannon T., Créton-Cazanave L., Dekens J., Fordham M., Gilbert C., Hewitt K, Kelman I., Morin J., N’Diaye A., O’Keefe P., Oliver-Smith A., Quesada C., Revet S., Sudmeier-Rieux K., Texier P., Vallette C., 2010. Alternatives pour une réduction durable des risques de catastrophe. Human Geography, vol. 3, n 1, p. 66-88.

Gustin P., 2017. Repenser les îles du Nord pour une reconstruction durable. Rapport de M. Philippe Gustin, Préfet, délégué interministériel à la reconstruction des îles de Saint-Barthélemy et de Saint-Martin. $34 \mathrm{p}$.

Hewitt K., 1983. Interpretations of Calamity From The Viewpoint of Human Ecology. Winchester, Allen and Unwin, $304 \mathrm{p}$.

Laguna Cruz J. A., Sánchez Arencibia A., 2006. La apreciación de los peligros de desastres en Cuba: tarea de toda la sociedad. Entorno géographico, $n^{\circ} 4$. Departemento de Géographia, Universidad del Valle, Cali (Colombia). 
Leone F., Vinet F. 2006. La vulnérabilité, un concept fondamental au cœur des méthodes d'évaluation des risques naturels. In Léone F., Vinet F. (dir.), 2006, La vulnérabilité des sociétés et des territoires face aux menaces naturelles. Analyses géographiques. Ed. de l'Université P. Valéry, Montpellier 3, p. 9-25.

Meléndez E., Hinojosa J. 2017. Estimates of Post-Hurricane Maria Exodus from Puerto Rico. Rapport du Centre for Puerto Rican Studies. New-York, Hunter College, CUNY.

Metzger P., D'Ercole R., 2011. Les risques en milieu urbain : éléments de réflexion. EchoGéo [En ligne], $\mathrm{n}^{\circ}$ 18. http://journals.openedition.org/echogeo/12640

Pasch R.J., Penny A.B., Berg R., 2018. Hurricane Maria, 16-30 September 2017 (AL 152017). National Hurricane Center Tropical cyclone Report, 48 p.

Pagney Bénito-Espinal F., 2003. Les ouragans Luis et Mitch et la pauvreté. Mappemonde $\mathrm{n}^{\circ} 72$, p. 44.

Pelling M. (dir.), 2003. Natural disasters and development in a globalizing world. Londres, Routledge, $268 \mathrm{p}$.

Pichler A., Striessnig E., 2013. Differential Vulnerability to Hurricanes in Cuba, Haiti, and the Dominican Republic: The Contribution of Education. Ecology and Society, vol. 18, n 3, p. 31.

Pielke R. et al., 2003. Hurricane Vulnerability in Latin America and The Caribbean: Normalized Damage and Loss Potentials. Natural Hazards Review, vol. 4, n 3, p. 101-114.

Quenault B., 2015. La résilience comme injonction politique post-Xynthia, EspacesTemps.net [En ligne], Travaux. https://www.espacestemps.net/articles/la-resilience-comme-injonctionpolitique-post-xynthia

Rappaport E., 2000. Loss of life in the United States associated with recent Atlantic tropical cyclones. Bulletin of American Meteorogical Society, $\mathrm{n}^{\circ}$ 81, p. 2065-2073.

Rappaport E., 2005. Addendum Huricane Andrew, 16-28 August 1992. NHC [En ligne]. https:// www.nhc.noaa.gov/1992andrew_add.html

Revet S., Langumier J. (dir.), 2013, Le gouvernement des catastrophes. Paris, Karthala, 280 p.

Rubiera J., 2014. Huracanes, cambio climático y reducción del riesgo. Temas, n 73, Enero-Marzo, La Habana, Cuba.

The Saffir-Simpson team, 2012. The Saffir-Simpson Hurricane wind scale, updated 1 February 2012 to reflect minor changes to category $3 / 4,4 / 5$ boundaries. Miami Florida, National Hurricane Center, $4 \mathrm{p}$.

Veyret Y., Reghezza M., 2006. Vulnérabilité et risques. L'approche récente de la vulnérabilité. Annales des Mines, $\mathrm{n}^{\circ}$ 43, p. 9-13.

Veyret Y., 2017. Une catastrophe inévitable: Saint-Martin (Antilles), une île à risques majeurs, Diploweb.com [En ligne]. https://www.diploweb.com/Une-catastrophe-inevitable-Saint-MartinAntilles-une-ile-a-risques-majeurs.html

\section{NOTES}

1. L'atterrissage d'un ouragan signifie l'arrivée sur les côtes de son centre (œil et mur de l'œil), donc de ses dangers maximaux. Selon la trajectoire de l'œil, il peut atterrir sur des territoires insulaires successifs. 
2. Le seuil de la catégorie 5 est estimé comme franchi à partir de vents moyens soutenus sur une minute de $252 \mathrm{~km} / \mathrm{h}$ soit 137 nœuds (knots ou kt).

3. L'échelle Saffir-Simpson d'intensité des ouragans a été révisée. Elle s'intitule désormais l'échelle de vent d'ouragan Saffir-Simpson et ne se base que sur les vents maximaux observés. En outre les limites des catégories $3 / 4$ et $4 / 5$ ont été légèrement revues (The Saffir-Simpson team, 2012).

4. Le classement de l'ouragan Andrew en catégorie 5 s'est fait a posteriori à partir notamment de la révision à la hausse de son impact sur les Bahamas et le sud de la Floride (Rappaport, addendum, 2005).

5. Les îles Vierges américaines et Porto Rico ont, au cours du XX $\mathrm{X}^{\mathrm{e}}$ siècle, été intégrés à des degrés divers aux États-Unis. Si les deux ensembles furent au départ des territoires « non incorporés » et " organisés », Porto Rico se distingua des îles Vierges en obtenant le statut d'État libre associé (Commonwealth of Puerto-Rico) en 1952.

6. Stéphane Pénet, directeur des assurances de dommages et de responsabilité de la Fédération française de l'assurance (FFA). In Compte rendu de la mission parlementaire sur la gestion des événements climatiques majeurs dans les zones littorales de l'Hexagone et des Outre-mer, CR n²7, mercredi 30 mai 2018, Assemblée Nationale.

7. Communiqué de la collectivité de Saint-Martin du 28 juillet 2018.

8. Rapport au Sénat américain de Charles R. Alexander Jr de l'USACE (U.S. Army Corps of Engineers) le 8 mai 2018. https://www.energy.senate.gov/public/index.cfm/files/serve? File_id=CD7A6805-9007-4D95-95E5-AC0CBA5A2CA2

9. Cf. https://rhg.com/research/puerto-rico-hurricane-maria-worlds-second-largest-blackout/ 10. Le cyclone Haiyan qui a sévèrement touché les Philippines en 2013 occupe la première place du classement. Id.

11. Le Président américain faisait ici référence aux 1833 morts liés au passage de Katrina dans le sud-ouest des États-Unis.

12. "Chaque mort est une horreur, mais si vous regardez une vraie catastrophe comme Katrina et vous regardez les énormes centaines et centaines de personnes qui sont mortes et ce qui s'est passé ici [...] Quel est votre bilan?», Discussion de Donald Trump avec les autorités locales le 3 octobre 2017. https://www.facebook.com/WhiteHouse/videos/1446951875392541/

13. Il faut ici noter une double difficulté d'ordre épistémologique. Il apparaît, d'une part, qu'un certain nombre de chercheurs se refusent à établir une distinction entre les victimes directes et indirectes à l'image de Kelman (2005), et, d'autre part, que la définition de la mortalité directe varie suivant les sources. Nous l'appréhendons comme les pertes en vies humaines qui ont lieu lorsque les aléas cycloniques exercent leurs effets y compris celles liées à des imprudences ou à une mauvaise appréciation du danger. Contrairement à certaines définitions en particulier celle du NHC (2018), nous y incluons les décès consécutifs à des attaques cardiaques, à des incendies de maisons, des électrocutions liées aux fils électriques arrachés, des accidents de voiture sur des routes détrempées dès lors qu'ils se produisent lors $\mathrm{du}$ passage du cyclone. Nous nous rapprochons ainsi de la définition donnée par E. Rappaport (2000) dans un article du bulletin de la société américaine de météorologie, qui voit la mortalité directe comme les fatalities attributable to the forces of the storms and their remnants.

14. Interview d'un membre de l'ODM (Office of Disaster Management) réalisée le 15 juin 2018 au siège de l'agence en charge de la gestion des catastrophes naturelles à Jimmit (Dominique).

15. Si l'on intègre la mortalité indirecte, le nombre de décès enregistrés suite au passage de Maria sur Porto Rico est bien plus élevé. Il serait de l'ordre de 3 à 4000 selon deux études parues en 2018. Kishore N. et al., 2018. Mortality in Puerto Rico after Hurricane Maria. New England journal of medicine, $\mathrm{n}^{\circ}$ 379, p. 162-170. Milken Institute School of Public Health, 2018. Ascertainment of the estimated excess mortality from hurricane María in Puerto Rico. George Washington University, Washington D.C., $11 \mathrm{p}$. 
16. Ces chiffres s'appuient sur les bilans officiels communiqués par les gouvernements et sur un redressement des données fournies par le rapport du NHC (2018) en fonction de la définition que nous donnons de la mortalité directe.

17. https://www.cia.gov/library/publications/the-world-factbook/rankorder/2004rank.html

18. Id.

19.

http://www.cerom-outremer.fr/IMG/pdf/note_cerom_pib_saint-barthelemy__octobre_2014.pdf

20. https://www.cia.gov/library/publications/the-world-factbook/rankorder/2004rank.html

21. L'Indice des risques climatiques (IRC) ne prend en compte que les événements liés à la météorologie: orages, inondations, excès thermiques. L'IRC est basé sur les chiffres de pertes enregistrées en 2016 et dans l'intervalle allant de 1996 à 2015. Le score de chaque pays dans l'Indice est fonction du nombre de décès, du nombre de décès par 100000 habitants, des pertes enregistrées en US\$ en pouvoir d'achat, ainsi que des pertes par unité du Produit intérieur brut, cf. Kreft S., Eckstein D., Melchior I., 2016. Indice des risques climatiques 2017. Qui souffre le plus des événements météorologiques extrêmes? Germanwatch, 8 p. [En ligne]. https:// germanwatch.org/fr/download/16413.pdf

22. La surinsularité désigne la situation de dépendance d'une île à l'égard d'une autre île. Cette situation décrite par P. Pelletier dans ses travaux sur le Japon se retrouve fréquemment au sein d'ensembles archipélagiques.

23. Voir à ce sujet l'article sur le site de la défense cubaine intitulé : «El ejercicio meteoro » : une experienca cubana [En ligne]. http://www.cubadefensa.cu/?q=ejercicio-meteoro

24. Selon le rapport du NHC concernant Irma (Cangialosi, 2018), 150000 maisons ont été endommagées et 15000 complètement détruites.

25. Au total, 150 pilleurs ont été interpellés le plus souvent a posteriori grâce aux images de caméras de surveillance.

\section{RÉSUMÉS}

Les auteurs s'intéressent dans cet article à l'impact des ouragans majeurs de la saison cyclonique 2017 sur la Caraibe insulaire, Irma et Maria. Ils soutiennent que le facteur principal de vulnérabilité des sociétés caribéennes n'est pas systématiquement lié à la puissance des ouragans ou au niveau de pauvreté que la récurrence de catastrophes en Haïti a contribué à fortement ancrer comme l'une des sources principales de vulnérabilité. En s'appuyant principalement sur les exemples cubain et saint-martinois, les auteurs montrent qu'il convient d'interroger les structures fondamentales des sociétés insulaires.

This article examines the impact of major hurricanes of the 2017 cyclonic season, Irma and Maria, on the the insular Caribbean region. The authors argue that the main factor of vulnerability of the caribbean societies is not systematically linked to the intensity of the hazard or the level of poverty that the recurrence of disasters in Haiti has helped to strongly anchor as one of the main sources of vulnerability. The analysis of the impacts of Irma and Maria on Cuba and Saint-Martin show that questions must be asked about the fundamental structures of island societies. 


\section{INDEX}

Mots-clés : ouragan, Caraïbe, bilan, pauvreté, fragilité sociale, vulnérabilité

Keywords : hurricane, Caribbean, assessment, poverty, social fragility, vulnerability

\section{AUTEURS}

\section{THIERRY NICOLAS}

Thierry Nicolas, thierrynicolas@wanadoo.fr, est maître de Conférences à l'Université de Guyane et membre de l'EA MINEA. Il a récemment publié :

- Nicolas T., 2016. Frontières, migrations et reconfigurations territoriales en Guyane. In Collomb G., Mam Lam Fouck S. (dir.), Mobilités, ethnicités, diversité culturelle : la Guyane entre Surinam et Brésil. Éléments de compréhension de la situation guyanaise. Matoury, Ibis Rouge Editions, p. 273-298.

- Lalubie G., Gros-Désormeaux J.-R., Nicolas T., 2015. L'application de la DCE dans les départements et régions d'outre-mer : la mise en lumière des discordances entre le concevable et le possible à la Martinique. Norois, ${ }^{\circ}$ 235, p. 51-66.

- Le Blanc A., Nicolas T. (dir.), 2013. Dossier « Politiques et pratiques de la résilience », EchoGéo [En ligne], $\mathrm{n}^{\circ} 24$. https://journals.openedition.org/echogeo/13451

\section{FRANÇOISE PAGNEY BÉNITO-ESPINAL}

Françoise Pagney Bénito-Espinal, Francoise.Pagney@gmail.com, est Professeur des Universités à l'Université des Antilles et membre de l'EA AIHP-GEODE.Elle a récemment publié :

- Pagney Bénito-Espinal F., 2015. Milieux et sociétés : un archipel à grande diversité écosystémique et forte emprise anthropique. In Huc J.C., Etna M. (dir.), Éclats de temps : Anthologie des événements extrêmes de la Guadeloupe. Gosier, PLB Éditions, p. 21-38.

- Pagney Bénito-Espinal F., Dumont J. (dir.), 2015. L'usage des plantes médicinales aux Antilles et en Guyane. Les remèdes anciens au fil du temps. Paris, Karthala, 288 p.

- Pagney Bénito-Espinal F., 2014. La mer Caraïbe : dynamique, enjeux passés présents et à venir. In Miossec A., Géographie des mers et des océans. Rennes, Presses universitaires de Rennes, p. $469-491$

\section{ÉMILIE LAGAHÉ}

Émilie Lagahé, emilie.lagahe@gmail.com, est ingénieure à l'Université des Antilles et membre de l'EA AIHP-GEODE. Elle a récemment publié :

- Leone F., Péroche M., Lagahé É., Gherardi M., Sahal A., Vinet F., Hachim S., Lavigne F., 2013. Modélisation de l'accessibilité territoriale pour l'aide à la gestion de crise tsunami (Mayotte, France). Annales de géographie, $\mathrm{n}^{\circ}$ 693, p. 502-524.

- Mellas S., Leone F., Omira R., Gherardi M. Baptista M.-A., Zourarah B., Péroche M., Lagahé É, 2012. Le risque tsunamique au Maroc : modélisation et évaluation au moyen d'un premier jeu d'indicateurs d'exposition du littoral atlantique. Physio-Géo [En ligne], Vol. 6. http:// journals.openedition.org/physio-geo/2589

\section{MARIE-LINE GOBINDDASS}

Marie-Line Gobinddass ml.gobinddass@gmail.com, est docteure en astronomie et astrophysique à l'Université de Guyane et membre de l'UMR ESPACE-DEV. Elle a récemment publié :

- Maingé P.E., Gobinddass M.-L., 2016. Convergence of one-step projected gradient methods for variational inequalities. Journal of Optimization Theory and Applications, Vol. 171, n 1, p 146-168. 
- Maingé P.E., Gobinddass M.-L., 2016. Convergence of one-step projected gradient methods for variational inequalities. Journal of Optimization Theory and Applications, Vol. 171, n 1, p 146-168.

- Marie-Joseph I., Linguet L., Gobinddass M.-L., Wald L., 2013. On the applicability of the

Heliosat-2 method to assess surface solar irradiance in the Intertropical Convergence Zone,

French Guiana. International Journal of Remote Sensing, vol. 34, n 8, p. 3012-3027. 